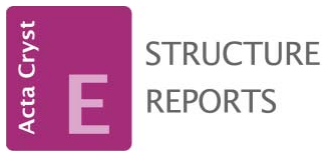

OPEN $\curvearrowright$ ACCESS

ISSN 1600-5368

\title{
Crystal structure of 2-tert-butyl-1,3-thia- zolo[4,5-b]pyridine
}

\author{
Gamal A. El-Hiti, ${ }^{a *}$ Keith Smith, ${ }^{b}$ Amany S. Hegazy, ${ }^{b}$ \\ Ali M. Masmali ${ }^{a}$ and Benson M. Kariuki ${ }^{b_{*}}$ \\ ${ }^{a}$ Cornea Research Chair, Department of Optometry, College of Applied Medical \\ Sciences, King Saud University, PO Box 10219, Riyadh 11433, Saudi Arabia, and \\ ${ }^{\mathbf{b}}$ School of Chemistry, Cardiff University, Main Building, Park Place, Cardiff \\ CF10 3AT, Wales. *Correspondence e-mail: gelhiti@ksu.edu.sa, \\ kariukib@cardiff.ac.uk
}

Received 2 July 2014; accepted 14 July 2014

Edited by G. Smith, Queensland University of Technology, Australia

The title compound, $\mathrm{C}_{10} \mathrm{H}_{12} \mathrm{~N}_{2} \mathrm{~S}$, does not contain any strong hydrogen-bond donors but two long $\mathrm{C}-\mathrm{H} \cdots \mathrm{N}$ contacts are observed in the crystal structure, with the most linear interaction linking molecules along [010]. The ellipsoids of the tert-butyl group indicate large librational motion.

Keywords: crystal structure; $\mathrm{C}-\mathrm{H} \cdots \mathrm{N}$ contacts; 1,3-thiazolo[4,5-b]pyriJpyridine.

CCDC reference: 1013859

\section{Related literature}

For the synthesis of substituted thiazolopyridines, see: Smith et al. (1994, 1995); El-Hiti (2003); Johnson et al. (2006); Rao et al. (2009); Sahasrabudhe et al. (2009); Lee et al. (2010); Chaban et al. (2013). For the crystal structure of a related compound, see: Yu et al. (2007).

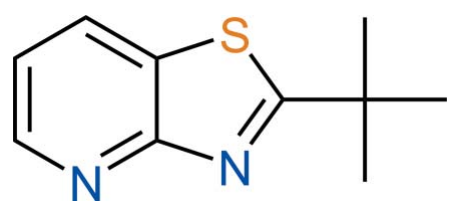

\section{Experimental}

\subsection{Crystal data}

$\mathrm{C}_{10} \mathrm{H}_{12} \mathrm{~N}_{2} \mathrm{~S}$

$M_{r}=192.28$

Orthorhombic, $P 2_{1} 2_{1} 2_{1}$

$a=9.4606$ (3) А

$b=9.7999(3) \AA$

$c=11.1155$ (4) ̊

$$
\begin{aligned}
& V=1030.55(6) \AA^{3} \\
& Z=4 \\
& \mathrm{Cu} K \alpha \text { radiation } \\
& \mu=2.42 \mathrm{~mm}^{-1} \\
& T=296 \mathrm{~K} \\
& 0.40 \times 0.29 \times 0.14 \mathrm{~mm}
\end{aligned}
$$

\subsection{Data collection \\ Agilent SuperNova (Dual, $\mathrm{Cu}$ at zero, Atlas) diffractometer \\ Absorption correction: multi-scan (CrysAlis PRO; Agilent, 2014) \\ $T_{\min }=0.721, T_{\max }=1.000$}

3395 measured reflections 1996 independent reflections 1951 reflections with $I>2 \sigma(I)$ $R_{\text {int }}=0.016$

\subsection{Refinement}

$R\left[F^{2}>2 \sigma\left(F^{2}\right)\right]=0.033$

$w R\left(F^{2}\right)=0.090$

$S=1.12$

1996 reflections

121 parameters

$\mathrm{H}$-atom parameters constrained

$\Delta \rho_{\max }=0.16{\mathrm{e} \AA^{-3}}^{-3}$

$\Delta \rho_{\min }=-0.22$ e $\AA^{-3}$

Absolute structure: Flack $x$ determined using 791 quotients $[(\mathrm{I}+)$ $(\mathrm{I}-)] /[(\mathrm{I}+)+(\mathrm{I}-)]$ (Parsons et al., 2013).

Absolute structure parameter: $0.027(7)$

Table 1

Hydrogen-bond geometry $\left(\AA{ }^{\circ}\right)$.

\begin{tabular}{lllll}
\hline$D-\mathrm{H} \cdots A$ & $D-\mathrm{H}$ & $\mathrm{H} \cdots A$ & $D \cdots A$ & $D-\mathrm{H} \cdots A$ \\
\hline $\mathrm{C} 4-\mathrm{H} 4 \cdots \mathrm{N} 1^{\mathrm{i}}$ & 0.93 & 2.81 & $3.564(3)$ & 138 \\
$\mathrm{C} 6-\mathrm{H} 6 \cdots \mathrm{N} 1^{\text {ii }}$ & 0.93 & 2.72 & $3.620(3)$ & 164 \\
\hline Symmetry codes: (i) $-x+\frac{3}{2},-y+1, z+\frac{1}{2} ;\left(\right.$ ii) $-x+1, y+\frac{1}{2},-z+\frac{1}{2}$. &
\end{tabular}

Data collection: CrysAlis PRO (Agilent, 2014); cell refinement: CrysAlis PRO; data reduction: CrysAlis PRO; program(s) used to solve structure: SHELXS2013 (Sheldrick, 2008); program(s) used to refine structure: SHELXL2013 (Sheldrick, 2008); molecular graphics: CHEMDRAW ultra (Cambridge Soft, 2001); software used to prepare material for publication: SHELXTL (Sheldrick, 2008).

\section{Acknowledgements}

The authors extend their appreciation to the Cornea Research Chair, Department of Optometry, College of Applied Medical Sciences, King Saud University, for funding this research.

Supporting information for this paper is available from the IUCr electronic archives (Reference: ZS2307).

\section{References}

Agilent (2014). CrysAlis PRO. Agilent Technologies, Yarnton, Oxfordshire, England.

Cambridge Soft (2001). CHEMDRAW Ultra. Cambridge Soft Corporation, Cambridge, Massachusetts, USA.

Chaban, T. I., Ogurtsov, V. V., Chaban, I. G., Klenina, O. V. \& Komarytsia, J. D. (2013). Phosphorus Sulfur Silicon Relat. Elem. 188, 1611-1620.

El-Hiti, G. A. (2003). Monatsh. Chem. 134, 837-841.

Johnson, S. G., Connolly, P. J. \& Murray, W. V. (2006). Tetrahedron Lett. 47, 4853-4856.

Lee, T., Lee, D., Lee, I. Y. \& Gong, Y.-D. (2010). J. Combin. Chem. 12, 95-99.

Parsons, S., Flack, H. D. \& Wagner, T. (2013). Acta Cryst. B69, 249-259.

Rao, A. U., Palani, A., Chen, X., Huang, Y., Aslanian, R. G., West, R. E. Jr, Williams, S. M., Wu, R.-L., Hwa, J., Sondey, C. \& Lachowicz, J. (2009). Bioorg. Med. Chem. Lett. 19, 6176-6180.

Sahasrabudhe, K. P., Estiarte, M. A., Tan, D., Zipfel, S., Cox, M., O'Mahony, D. J. R., Edwards, W. T. \& Duncton, M. A. J. (2009). J. Heterocycl. Chem. 46, $1125-1131$.

Sheldrick, G. M. (2008). Acta Cryst. A64, 112-122.

Smith, K., Anderson, D. \& Matthews, I. (1995). Sulfur Lett. 18, 79-95.

Smith, K., Lindsay, C. M., Morris, I. K., Matthews, I. \& Pritchard, G. J. (1994). Sulfur Lett. 17, 197-216.

Yu, Y.-Q., Wang, Y., Ni, P.-Z. \& Lu, T. (2007). Acta Cryst. E63, o968-o969. 


\title{
supporting information
}

Acta Cryst. (2014). E70, o932 [doi:10.1107/S160053681401633X]

\section{Crystal structure of 2-tert-butyl-1,3-thiazolo[4,5-b]pyridine}

\author{
Gamal A. El-Hiti, Keith Smith, Amany S. Hegazy, Ali M. Masmali and Benson M. Kariuki
}

\section{S1. Structural commentary}

Various methods have been reported for the synthesis of substituted thiazolopyridines (Smith et al., 1994, 1995; El-Hiti, 2003; Johnson et al., 2006; Rao et al., 2009; Sahasrabudhe et al., 2009; Lee et al., 2010; Chaban et al., 2013). In a continuation of our research focused on new synthetic routes towards substituted heterocycles we have synthesized the title compound 2-tert-butylthiazolo[4,5-b]pyridine in high yield (Smith et al., 1995; El-Hiti, 2003) and now report its Xray crystal structure. The X-ray structure for a related compound has been reported previously (Yu et al., 2007). In the title compound (Fig. 1), the ellipsoids of the methyl groups of the tert-butyl group are large which is consistent with librational motion of the group. Assumption of a disordered model did not show significant improvement in the refinement. The molecule does not contain strong hydrogen bond donors. In the crystal, two long $\mathrm{C}-\mathrm{H} \cdots \mathrm{N}$ contacts are observed, the most linear of which links the molecules in chains along [010] (Fig. 2).

\section{S2. Synthesis and crystallization}

2-tert-Butylthiazolo[4,5-b]pyridine was obtained in 97\% yield from acid hydrolysis of 3-(diisopropylaminothiocarbonylthio)-2-(pivalamido)pyridine under reflux (Smith et al., 1995). The compound may also be synthesized in 66\% yield from reaction of 3-(diisopropylaminothiocarbonylthio)-2-aminopyridine with 2,2-dimethylpropionic acid in the presence of phosphorus oxychloride under reflux (El-Hiti, 2003). Crystallization from a mixture of ethyl acetate and diethyl ether (1:3 by volume) gave the title compound as colourless crystals. The NMR and low and high resolution mass spectra for the title compound were consistent with those previously reported (Smith et al., 1995).

\section{S3. Refinement}

Crystal data, data collection and structure refinement details are summarized in the crystal data table. The hydrogen atoms were positioned geometrically and refined using a riding model with $U_{\text {iso }}(\mathrm{H})=1.2$ times $U_{\text {eq }}$ for the atom to which they are bonded in the case of aromatic rings, $\mathrm{NH}$ and $\mathrm{CH}_{2}$ groups and 1.5 times $U_{\text {eq }}$ for the methyl hydrogens. Crystal data, data collection and structure refinement details are summarized in Table 1. The Flack parameter (Parsons et al., 2013) was 0.027 (7) but is not of any structural relevance with this compound. 


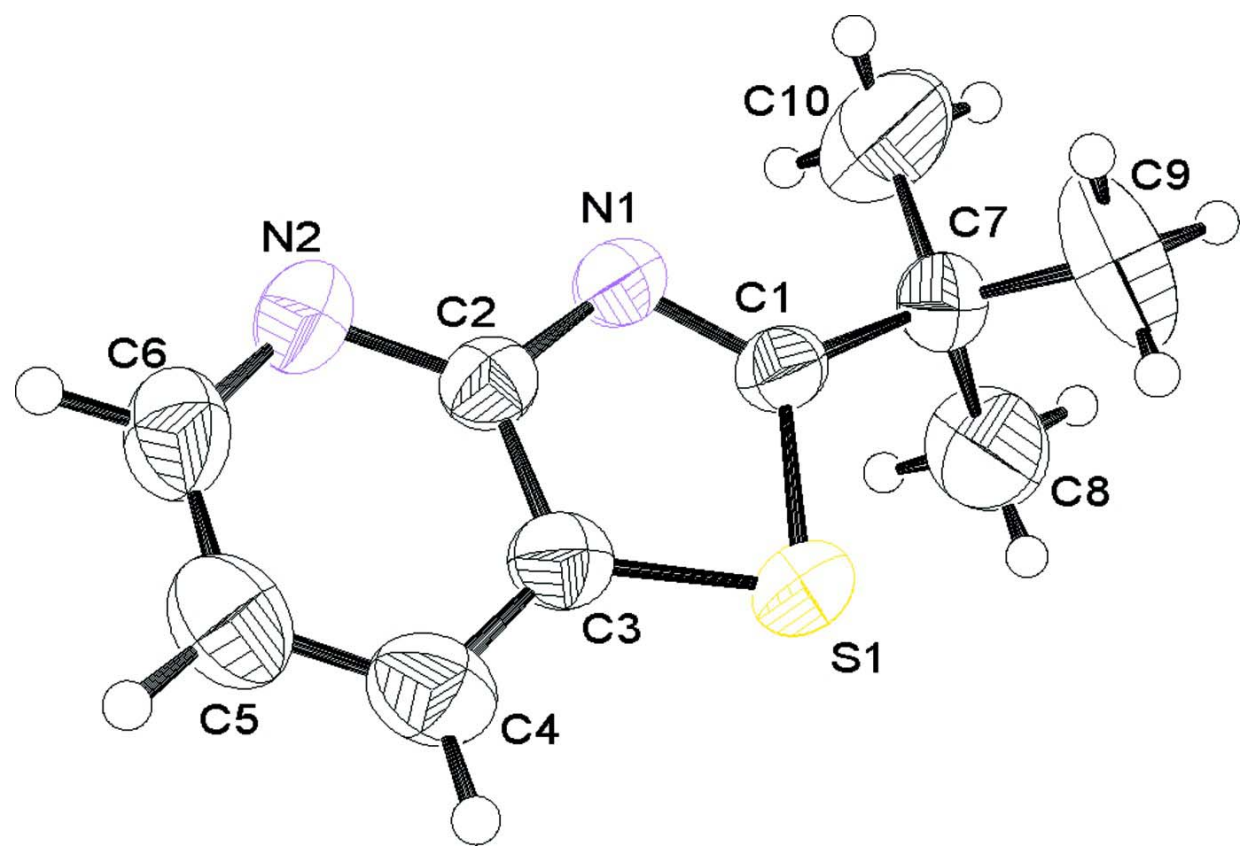

\section{Figure 1}

A molecule of the title compound showing atom labels and 50\% probability displacement ellipsoids for non- $\mathrm{H}$ atoms.

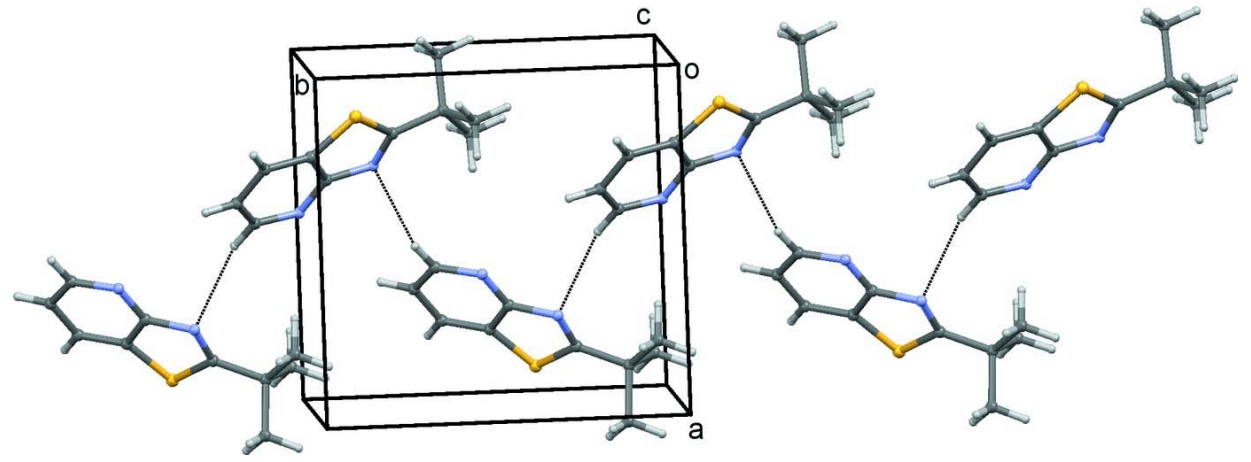

Figure 2

Crystal structure packing with the long linear $\mathrm{C}-\mathrm{H} \cdots \mathrm{N}$ contacts shown as dashed lines.

\section{2-tert-Butyl-1,3-thiazolo[4,5-b]pyridine}

\section{Crystal data}

$\mathrm{C}_{10} \mathrm{H}_{12} \mathrm{~N}_{2} \mathrm{~S}$

$M_{r}=192.28$

Orthorhombic, $P 22_{1} 2_{1} 2_{1}$

$a=9.4606$ (3) $\AA$

$b=9.7999(3) \AA$

$c=11.1155$ (4) $\AA$

$V=1030.55(6) \AA^{3}$

$Z=4$

$F(000)=408$
$D_{\mathrm{x}}=1.239 \mathrm{Mg} \mathrm{m}^{-3}$

$\mathrm{Cu} K \alpha$ radiation, $\lambda=1.54184 \AA$

Cell parameters from 1951 reflections

$\theta=6.0-73.4^{\circ}$

$\mu=2.42 \mathrm{~mm}^{-1}$

$T=296 \mathrm{~K}$

Plate, colourless

$0.40 \times 0.29 \times 0.14 \mathrm{~mm}$ 


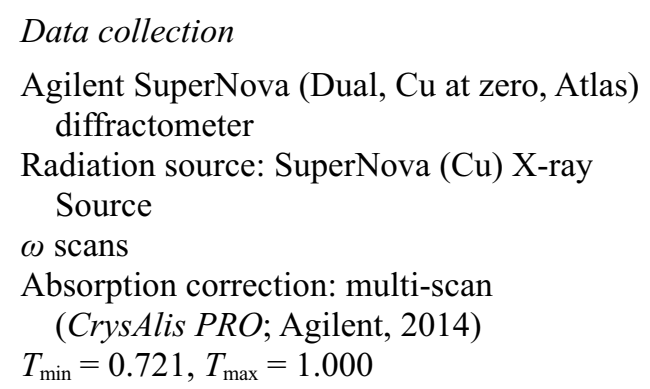

Refinement

Refinement on $F^{2}$

Least-squares matrix: full

$R\left[F^{2}>2 \sigma\left(F^{2}\right)\right]=0.033$

$w R\left(F^{2}\right)=0.090$

$S=1.12$

1996 reflections

121 parameters

0 restraints

Hydrogen site location: inferred from

neighbouring sites

$$
\begin{aligned}
& 3395 \text { measured reflections } \\
& 1996 \text { independent reflections } \\
& 1951 \text { reflections with } I>2 \sigma(I) \\
& R_{\text {int }}=0.016 \\
& \theta_{\max }=73.4^{\circ}, \theta_{\min }=6.0^{\circ} \\
& h=-7 \rightarrow 11 \\
& k=-11 \rightarrow 12 \\
& l=-13 \rightarrow 10
\end{aligned}
$$

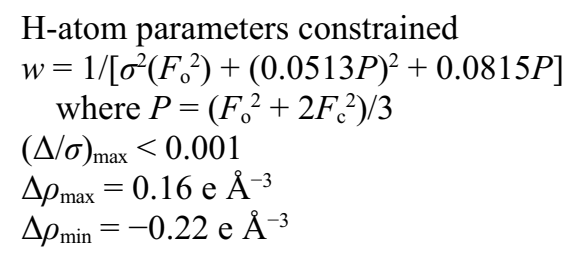

Absolute structure: Flack $x$ determined using 791 quotients $[(\mathrm{I}+)-(\mathrm{I}-)] /[(\mathrm{I}+)+(\mathrm{I}-)]$ (Parsons et al., 2013).

Absolute structure parameter: 0.027 (7)

Special details

Geometry. All e.s.d.'s (except the e.s.d. in the dihedral angle between two 1.s. planes) are estimated using the full covariance matrix. The cell e.s.d.'s are taken into account individually in the estimation of e.s.d.'s in distances, angles and torsion angles; correlations between e.s.d.'s in cell parameters are only used when they are defined by crystal symmetry. An approximate (isotropic) treatment of cell e.s.d.'s is used for estimating e.s.d.'s involving 1.s. planes.

Fractional atomic coordinates and isotropic or equivalent isotropic displacement parameters $\left(\AA^{2}\right)$

\begin{tabular}{lllll}
\hline & $x$ & $y$ & $z$ & $U_{\text {iso }} * U_{\text {eq }}$ \\
\hline C1 & $0.8245(2)$ & $0.2846(2)$ & $0.37042(19)$ & $0.0458(5)$ \\
C2 & $0.6967(2)$ & $0.4685(2)$ & $0.33748(19)$ & $0.0435(5)$ \\
C3 & $0.7716(2)$ & $0.5154(3)$ & $0.4378(2)$ & $0.0483(5)$ \\
C4 & $0.7455(3)$ & $0.6454(3)$ & $0.4822(3)$ & $0.0645(6)$ \\
H4 & 0.7924 & 0.6789 & 0.5496 & $0.077^{*}$ \\
C5 & $0.6470(3)$ & $0.7218(3)$ & $0.4218(3)$ & $0.0677(7)$ \\
H5 & 0.6260 & 0.8097 & 0.4477 & $0.081^{*}$ \\
C6 & $0.5791(3)$ & $0.6682(3)$ & $0.3225(3)$ & $0.0656(7)$ \\
H6 & 0.5138 & 0.7234 & 0.2833 & $0.079^{*}$ \\
C7 & $0.8831(3)$ & $0.1417(2)$ & $0.3577(2)$ & $0.0556(6)$ \\
C8 & $0.8599(5)$ & $0.0641(4)$ & $0.4747(3)$ & $0.0938(11)$ \\
H8A & 0.9096 & 0.1090 & 0.5387 & $0.141^{*}$ \\
H8B & 0.8948 & -0.0274 & 0.4662 & $0.141^{*}$ \\
H8C & 0.7608 & 0.0617 & 0.4929 & $0.141^{*}$ \\
C9 & $1.0411(4)$ & $0.1506(5)$ & $0.3319(5)$ & $0.1151(16)$ \\
H9A & 1.0560 & 0.1983 & 0.2575 & $0.173^{*}$ \\
H9B & 1.0798 & 0.0603 & 0.3260 & $0.173^{*}$ \\
H9C & 1.0871 & 0.1990 & 0.3961 & $0.173^{*}$ \\
C10 & $0.8067(6)$ & $0.0657(4)$ & $0.2577(4)$ & $0.124(2)$ \\
H10A & 0.7076 & 0.0614 & 0.2758 & $0.186^{*}$
\end{tabular}




\begin{tabular}{lllll} 
H10B & 0.8440 & -0.0251 & 0.2513 & $0.186^{*}$ \\
H10C & 0.8203 & 0.1129 & 0.1829 & $0.186^{*}$ \\
N1 & $0.72864(19)$ & $0.3364(2)$ & $0.30173(16)$ & $0.0453(4)$ \\
N2 & $0.6004(2)$ & $0.5424(2)$ & $0.2787(2)$ & $0.0576(5)$ \\
S1 & $0.88585(7)$ & $0.39028(7)$ & $0.48644(5)$ & $0.0607(2)$ \\
\hline
\end{tabular}

Atomic displacement parameters $\left(\AA^{2}\right)$

\begin{tabular}{lllllll}
\hline & $U^{11}$ & $U^{22}$ & $U^{33}$ & $U^{12}$ & $U^{13}$ & $U^{23}$ \\
\hline $\mathrm{C} 1$ & $0.0431(10)$ & $0.0571(12)$ & $0.0371(10)$ & $0.0007(10)$ & $-0.0013(8)$ & $0.0016(9)$ \\
$\mathrm{C} 2$ & $0.0423(10)$ & $0.0483(11)$ & $0.0398(10)$ & $-0.0043(9)$ & $0.0022(8)$ & $0.0053(9)$ \\
$\mathrm{C} 3$ & $0.0443(11)$ & $0.0550(12)$ & $0.0457(11)$ & $-0.0048(9)$ & $0.0022(9)$ & $-0.0035(9)$ \\
$\mathrm{C} 4$ & $0.0664(15)$ & $0.0611(14)$ & $0.0661(15)$ & $-0.0075(11)$ & $0.0034(13)$ & $-0.0160(13)$ \\
$\mathrm{C} 5$ & $0.0707(17)$ & $0.0500(13)$ & $0.0823(19)$ & $-0.0008(12)$ & $0.0129(15)$ & $-0.0023(13)$ \\
$\mathrm{C} 6$ & $0.0686(16)$ & $0.0542(13)$ & $0.0740(17)$ & $0.0102(12)$ & $0.0041(14)$ & $0.0157(13)$ \\
$\mathrm{C} 7$ & $0.0564(12)$ & $0.0560(13)$ & $0.0544(12)$ & $0.0118(11)$ & $-0.0010(11)$ & $0.0012(10)$ \\
$\mathrm{C} 8$ & $0.122(3)$ & $0.078(2)$ & $0.081(2)$ & $0.0248(19)$ & $0.009(2)$ & $0.0219(17)$ \\
$\mathrm{C} 9$ & $0.074(2)$ & $0.097(3)$ & $0.174(5)$ & $0.0241(19)$ & $0.044(3)$ & $-0.007(3)$ \\
$\mathrm{C} 10$ & $0.187(5)$ & $0.073(2)$ & $0.112(3)$ & $0.052(3)$ & $-0.072(3)$ & $-0.034(2)$ \\
$\mathrm{N} 1$ & $0.0468(9)$ & $0.0492(9)$ & $0.0401(9)$ & $0.0001(8)$ & $-0.0045(8)$ & $0.0005(8)$ \\
$\mathrm{N} 2$ & $0.0588(11)$ & $0.0570(11)$ & $0.0570(12)$ & $0.0070(10)$ & $-0.0078(10)$ & $0.0101(9)$ \\
S1 & $0.0568(3)$ & $0.0761(4)$ & $0.0493(3)$ & $0.0096(3)$ & $-0.0161(3)$ & $-0.0112(3)$ \\
\hline
\end{tabular}

Geometric parameters $\left(\AA,{ }^{\circ}\right)$

\begin{tabular}{llll}
\hline $\mathrm{C} 1-\mathrm{N} 1$ & $1.290(3)$ & $\mathrm{C} 6-\mathrm{H} 6$ & 0.9300 \\
$\mathrm{C} 1-\mathrm{C} 7$ & $1.512(3)$ & $\mathrm{C} 7-\mathrm{C} 10$ & $1.521(4)$ \\
$\mathrm{C} 1-\mathrm{S} 1$ & $1.753(2)$ & $\mathrm{C} 7-\mathrm{C} 8$ & $1.522(4)$ \\
$\mathrm{C} 2-\mathrm{N} 2$ & $1.335(3)$ & $\mathrm{C} 7-\mathrm{C} 9$ & $1.524(4)$ \\
$\mathrm{C} 2-\mathrm{N} 1$ & $1.387(3)$ & $\mathrm{C} 8-\mathrm{H} 8 \mathrm{~A}$ & 0.9600 \\
$\mathrm{C} 2-\mathrm{C} 3$ & $1.399(3)$ & $\mathrm{C} 8-\mathrm{H} 8 \mathrm{~B}$ & 0.9600 \\
$\mathrm{C} 3-\mathrm{C} 4$ & $1.389(4)$ & $\mathrm{C} 8-\mathrm{H} 8 \mathrm{C}$ & 0.9600 \\
$\mathrm{C} 3-\mathrm{S} 1$ & $1.722(3)$ & $\mathrm{C} 9-\mathrm{H} 9 \mathrm{~A}$ & 0.9600 \\
$\mathrm{C} 4-\mathrm{C} 5$ & $1.371(4)$ & $\mathrm{C} 9-\mathrm{H} 9 \mathrm{~B}$ & 0.9600 \\
$\mathrm{C} 4-\mathrm{H} 4$ & 0.9300 & $\mathrm{C} 9-\mathrm{H} 9 \mathrm{C}$ & 0.9600 \\
$\mathrm{C} 5-\mathrm{C} 6$ & $1.380(4)$ & $\mathrm{C} 10-\mathrm{H} 10 \mathrm{~A}$ & 0.9600 \\
$\mathrm{C} 5-\mathrm{H} 5$ & 0.9300 & $\mathrm{C} 10-\mathrm{H} 10 \mathrm{~B}$ & 0.9600 \\
$\mathrm{C} 6-\mathrm{N} 2$ & $1.341(4)$ & $\mathrm{C} 10-\mathrm{H} 10 \mathrm{C}$ & 0.9600 \\
& & & \\
$\mathrm{~N} 1-\mathrm{C} 1-\mathrm{C} 7$ & $124.6(2)$ & $\mathrm{C} 8-\mathrm{C} 7-\mathrm{C} 9$ & $109.3(3)$ \\
$\mathrm{N} 1-\mathrm{C} 1-\mathrm{S} 1$ & $115.80(18)$ & $\mathrm{C} 7-\mathrm{C} 8-\mathrm{H} 8 \mathrm{~A}$ & 109.5 \\
$\mathrm{C} 7-\mathrm{C} 1-\mathrm{S} 1$ & $119.64(17)$ & $\mathrm{C} 7-\mathrm{C} 8-\mathrm{H} 8 \mathrm{~B}$ & 109.5 \\
$\mathrm{~N} 2-\mathrm{C} 2-\mathrm{N} 1$ & $121.0(2)$ & $\mathrm{H} 8 \mathrm{~A}-\mathrm{C} 8-\mathrm{H} 8 \mathrm{~B}$ & 109.5 \\
$\mathrm{~N} 2-\mathrm{C} 2-\mathrm{C} 3$ & $123.9(2)$ & $\mathrm{C} 7-\mathrm{C} 8-\mathrm{H} 8 \mathrm{C}$ & 109.5 \\
$\mathrm{~N} 1-\mathrm{C} 2-\mathrm{C} 3$ & $115.1(2)$ & $\mathrm{H} 8 \mathrm{~A}-\mathrm{C} 8-\mathrm{H} 8 \mathrm{C}$ & 109.5 \\
$\mathrm{C} 4-\mathrm{C} 3-\mathrm{C} 2$ & $119.7(2)$ & $\mathrm{H} 8 \mathrm{~B}-\mathrm{C} 8-\mathrm{H} 8 \mathrm{C}$ & 109.5 \\
$\mathrm{C} 4-\mathrm{C} 3-\mathrm{S} 1$ & $130.8(2)$ & $\mathrm{C} 7-\mathrm{C} 9-\mathrm{H} 9 \mathrm{~A}$ & 109.5 \\
$\mathrm{C} 2-\mathrm{C} 3-\mathrm{S} 1$ & $109.55(18)$ & $\mathrm{C} 7-\mathrm{C} 9-\mathrm{H} 9 \mathrm{~B}$ &
\end{tabular}


supporting information

$\begin{array}{llll}\mathrm{C} 5-\mathrm{C} 4-\mathrm{C} 3 & 116.6(3) & \mathrm{H} 9 \mathrm{~A}-\mathrm{C} 9-\mathrm{H} 9 \mathrm{~B} & 109.5 \\ \mathrm{C} 5-\mathrm{C} 4-\mathrm{H} 4 & 121.7 & \mathrm{C} 7-\mathrm{C} 9-\mathrm{H} 9 \mathrm{C} & 109.5 \\ \mathrm{C} 3-\mathrm{C} 4-\mathrm{H} 4 & 121.7 & \mathrm{H} 9 \mathrm{~A}-\mathrm{C} 9-\mathrm{H} 9 \mathrm{C} & 109.5 \\ \mathrm{C} 4-\mathrm{C} 5-\mathrm{C} 6 & 120.0(3) & \mathrm{H} 9 \mathrm{~B}-\mathrm{C} 9-\mathrm{H} 9 \mathrm{C} & 109.5 \\ \mathrm{C} 4-\mathrm{C} 5-\mathrm{H} 5 & 120.0 & \mathrm{C}-\mathrm{C} 10-\mathrm{H} 10 \mathrm{~A} & 109.5 \\ \mathrm{C} 6-\mathrm{C} 5-\mathrm{H} 5 & 120.0 & \mathrm{C} 7-\mathrm{C} 10-\mathrm{H} 10 \mathrm{~B} & 109.5 \\ \mathrm{~N} 2-\mathrm{C} 6-\mathrm{C} 5 & 124.8(3) & \mathrm{H} 10 \mathrm{~A}-\mathrm{C} 10-\mathrm{H} 10 \mathrm{~B} & 109.5 \\ \mathrm{~N} 2-\mathrm{C} 6-\mathrm{H} 6 & 117.6 & \mathrm{C} 7-\mathrm{C} 10-\mathrm{H} 10 \mathrm{C} & 109.5 \\ \mathrm{C} 5-\mathrm{C} 6-\mathrm{H} 6 & 117.6 & \mathrm{H} 10 \mathrm{~A}-\mathrm{C} 10-\mathrm{H} 10 \mathrm{C} & 109.5 \\ \mathrm{C} 1-\mathrm{C} 7-\mathrm{C} 10 & 110.3(2) & \mathrm{H} 10 \mathrm{~B}-\mathrm{C} 10-\mathrm{H} 10 \mathrm{C} & 109.5 \\ \mathrm{C} 1-\mathrm{C} 7-\mathrm{C} 8 & 109.3(2) & \mathrm{C} 1-\mathrm{N} 1-\mathrm{C} 2 & 110.6(2) \\ \mathrm{C} 10-\mathrm{C} 7-\mathrm{C} 8 & 108.1(3) & \mathrm{C} 2-\mathrm{N} 2-\mathrm{C} 6 & 115.0(2) \\ \mathrm{C} 1-\mathrm{C} 7-\mathrm{C} 9 & 108.9(3) & \mathrm{C} 3-\mathrm{S} 1-\mathrm{C} 1 & 88.96(11) \\ \mathrm{C} 10-\mathrm{C} 7-\mathrm{C} 9 & 110.9(3) & & \end{array}$

Hydrogen-bond geometry $\left(\AA,{ }^{\circ}\right)$

\begin{tabular}{lllll}
\hline$D-\mathrm{H} \cdots A$ & $D-\mathrm{H}$ & $\mathrm{H} \cdots A$ & $D \cdots A$ & $D-\mathrm{H} \cdots A$ \\
\hline $\mathrm{C} 4-\mathrm{H} 4 \cdots \mathrm{N} 1^{\mathrm{i}}$ & 0.93 & 2.81 & $3.564(3)$ & 138 \\
$\mathrm{C} 6-\mathrm{H} 6 \cdots \mathrm{N} 1^{\mathrm{ii}}$ & 0.93 & 2.72 & $3.620(3)$ & 164 \\
\hline
\end{tabular}

Symmetry codes: (i) $-x+3 / 2,-y+1, z+1 / 2$; (ii) $-x+1, y+1 / 2,-z+1 / 2$. 